\title{
Pancreatic metastasis from phyllodes tumor presenting initially as acute retroperitoneal hemorrhage
}

\author{
Masao Yukawa $\cdot$ Masahiro Watatani · \\ Sayuri Isono $\cdot$ Hiroyuki Shiono $\cdot$ Hirokazu Hasegawa • \\ Kaoru Okajima $\cdot$ Yoshio Ohta $\cdot$ Masatoshi Inoue
}

Received: 17 January 2013/Accepted: 10 March 2013/Published online: 27 March 2013

(C) The Japan Society of Clinical Oncology 2013

\begin{abstract}
Metastasis from mammary phyllodes tumor is rare; only five cases of phyllodes tumor metastasizing to the pancreas have been documented in the literature. Our patient developed acute retroperitoneal hemorrhage due to pancreatic metastasis from malignant phyllodes tumor of the breast, as the first presentation of metastasis. We successfully used catheter intervention to avoid urgent explorative laparotomy, and eventually found another metastasis in a short period. Systemic ifosfamide-doxorubicin combination chemotherapy was employed. The regression of metastases was partial and a new lesion appeared at the same time. This case is extremely rare. However, we should be careful about the symptoms indicating the sign of bleeding during the follow-up of patients with fast-progressing metastatic phyllodes tumor.
\end{abstract}

Keywords Phyllodes tumor · Pancreatic metastasis · Retroperitoneal hemorrhage $\cdot$ TAE $\cdot$ Ifosfamide

M. Yukawa $(\bowtie) \cdot$ M. Watatani $\cdot$ S. Isono $\cdot$ M. Inoue Department of Surgery, Kinki University-Faculty of Medicine, Nara Hospital, 1248-1 Otoda-Cho, Ikoma, Nara, Japan e-mail: yukawa@nara.med.kindai.ac.jp

H. Shiono

Department of General Thoracic Surgery, Kinki UniversityFaculty of Medicine, Nara Hospital, 1248-1 Otoda-Cho, Ikoma, Nara, Japan

H. Hasegawa $\cdot$ K. Okajima

Department of Radiology, Kinki University-Faculty of Medicine, Nara Hospital, 1248-1 Otoda-Cho, Ikoma, Nara, Japan

\section{Y. Ohta}

Department of Pathology and Laboratory Medicine, Kinki University-Faculty of Medicine, Nara Hospital, 1248-1 Otoda-Cho, Ikoma, Nara, Japan

\section{Introduction}

Phyllodes tumor (PT) is a rare, biphasic, fibroepithelial neoplasm of the breast that usually occurs in middle-aged women. It constitutes $0.3 \%$ of breast tumors. PT can be further classified as benign, borderline, or malignant on the basis of its histologic characteristics [1-4]. Distant metastasis occurs in approximately $25 \%$ of malignant PT [2]. The most common sites of distant metastases are the lungs [3, 5]. Metastases to the pancreas are extremely rare [6-10]. Here, for the first time, we report a case of PT-derived pancreatic metastasis presenting as retroperitoneal hemorrhage, which was controlled with catheter intervention.

\section{Case report}

The patient was a 51-year-old woman, who had undergone a modified radical mastectomy with a transverse rectus abdominus musculocutaneous flap for a very large PT in her left breast (Fig. 1). The resected tumor was $12 \mathrm{~cm}$ in diameter. The cut surface was solid, gray-white, and showed cleft-like spaces (Fig. 1c). Microscopically, HE staining showed a well-circumscribed biphasic neoplasm, consisting mainly of stromal component with benign glandular elements (Fig. 2a). Stromal component showed various degrees of cellularity and nuclear atypia from area to area. Some areas showed marked proliferation of atypical spindle cells with stromal overgrowth; the mitotic activity was 48 mitotic figures per 10 high power fields (Fig. 2b). The overall appearance was consistent with the diagnosis of malignant PT. She received no adjuvant therapy. Fifteen months after the surgery, she visited our hospital with a week's history of back pain, which had acutely worsened during the visit. She had no symptoms at 
Fig. 1 CT of primary phyllodes tumor. a 3D reconstruction shows a very large lump on left breast. b Cross section of the tumor shows heterogeneous profile. c Macroscopic appearance of surgical specimen: cut surface
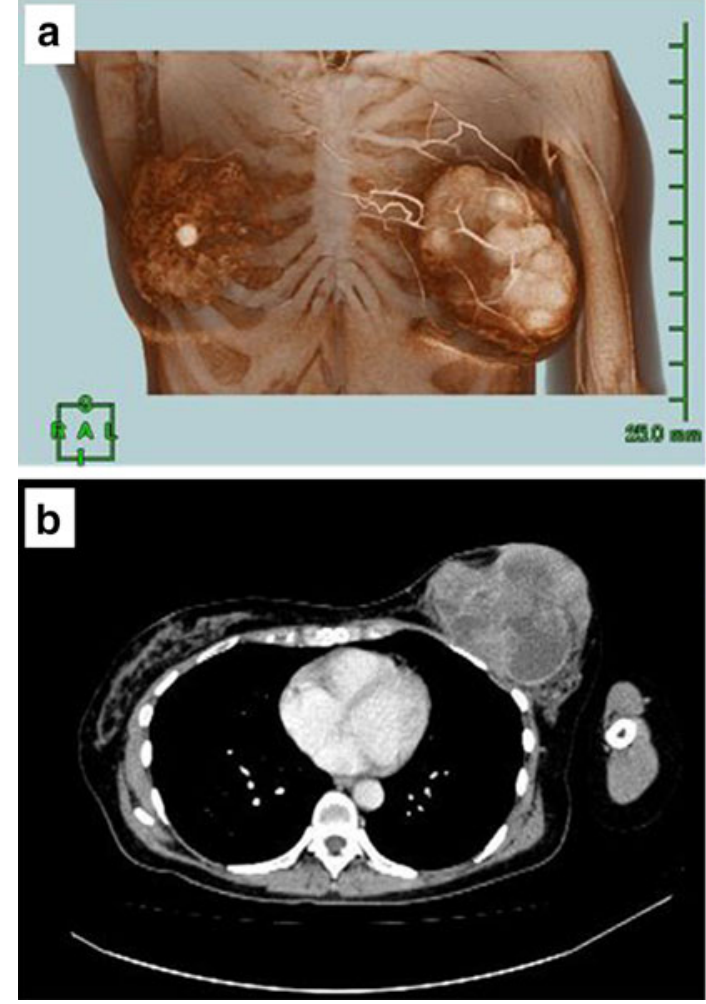

the last visit, 3 months previous; periodic examinations, including abdominal ultrasonography (US), showed no evidence of metastasis.

On admission to our hospital, she had an enhanced computed tomography (CT) examination, which revealed a retroperitoneal hemorrhage and hematoma, with a tumor in the pancreatic tail (Fig. 3). Urgent angiography showed active bleeding from a splenic artery branch (Fig. 4). Transarterial selective embolization was performed, which controlled the bleeding and stabilized the patient's condition. However, rebleeding was found the next day, and a second embolization was performed. We ultimately needed three embolizations for successful hemostasis, including an embolus of a splenic artery.

Sixteen days later, a follow-up CT after embolization disclosed new tumors in the liver and in the lung (Fig. 5). Because the new lung and liver tumors appeared very shortly after the pancreas tumor, we regarded these tumors as metastases from the same origin. A pulmonary tumor was biopsied by video-assisted thoracic surgery (VATS). Microscopically, the tumor showed marked proliferation of atypical spindle cells with a mitotic activity of greater than 40 mitotic figures per 10 high power fields, which mimicked the malignant part of the primary tumor; pathological diagnosis was a metastasis from a malignant PT (Fig. 6). Thus, we concluded that the pancreas tumor and these tumors were metastases of the malignant breast PT. We decided to treat her with systemic chemotherapy. Five weeks after the biopsy, the metastases in the pancreas, liver, and lung showed very rapid growth prior to chemotherapy (Fig. 7, top).

She was treated with ifosfamide-doxorubicin combination chemotherapy (doxorubicin $25 \mathrm{mg} / \mathrm{m}^{2} /$ day, days $1-2$; ifosfamide $2,000 \mathrm{mg} / \mathrm{m}^{2} /$ day, days $\left.1-5\right)$. Although the follow-up CT after chemotherapy showed partial regression of liver and pancreas metastasis (Fig. 7, bottom), she refused further chemotherapy. She opted for best supportive care at home, and died a month later.

\section{Discussion}

From 2001 to 2011, we treated 76 patients with fibroepithelial tumors that were greater than $3 \mathrm{~cm}$ in diameter or growing very rapidly, in accordance with the policy of Noguchi et al. [4]. Of these, 12 cases were PT; four of these 12 were diagnosed as malignant PT. The present case was the first in our institute to develop distant metastases.

PT constitutes less than $1 \%$ of all breast tumors and $2.5 \%$ of breast fibroepithelial tumors. PT comprises a benign epithelial element and a monoclonal stromal cell component [1-4]. PT is further classified as benign, borderline, or malignant on the basis of features of the stromal cells; $10-20 \%$ of PT are classified as malignant [1, 2]. Distant metastasis occurs with approximately $25 \%$ of malignant PT [2]. The most common sites of distant metastases are the lungs $[3,5]$; metastases to the pancreas 

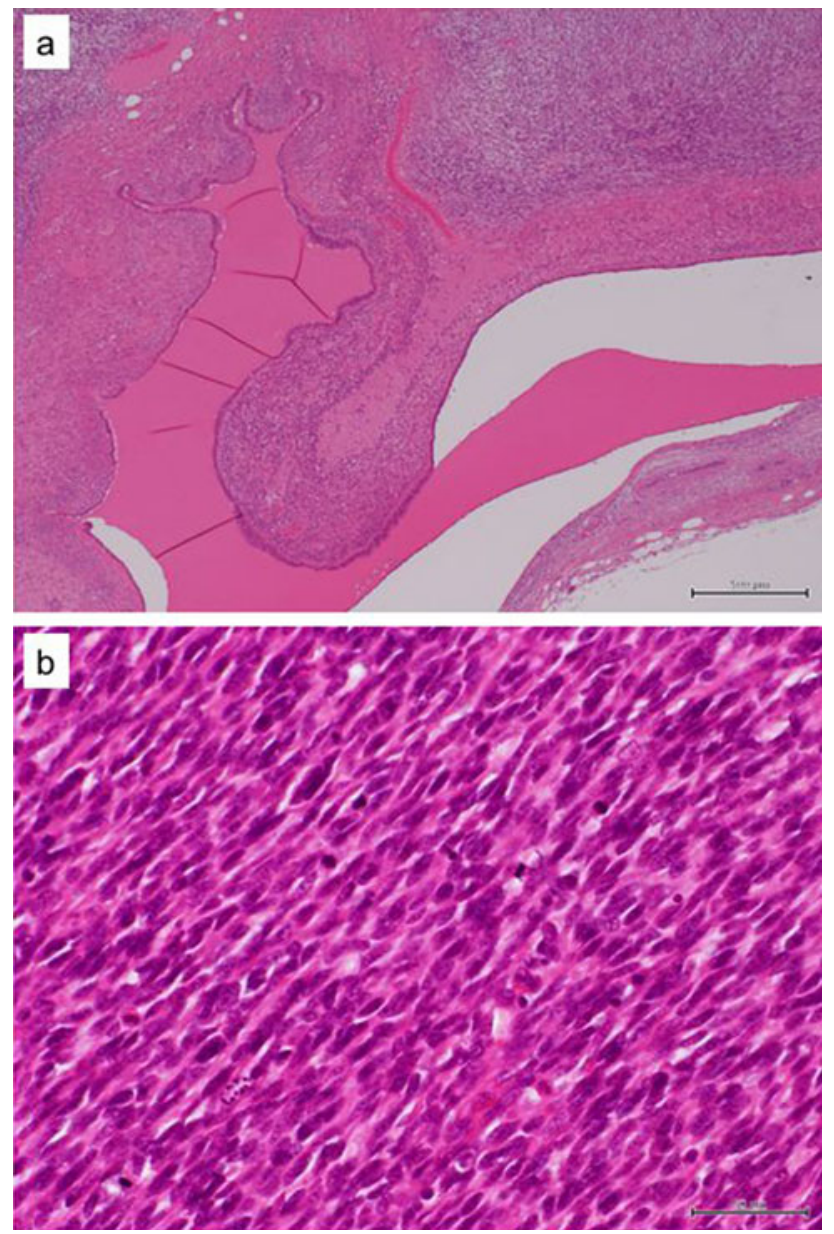

Fig. 2 Microscopic findings of primary tumor: low-power magnification (a) and high-power magnification (b). Primary tumor comprises stromal component and epithelial component showing intracanalicular pattern (a). Another area of primary tumor shows marked proliferation of spindle cells with numerous mitotic figures (b). Scale bars $500 \mu \mathrm{m}$ in $\mathbf{a}$ and $50 \mu \mathrm{m}$ in $\mathbf{b}$

from PT are extremely rare. A comprehensive review of the literature identified only five such case reports [6-10]; aside from one report of hemorrhage in the digestive tract [8], bleeding from pancreatic metastasis has not been described. In four of the five reported cases, pancreatic metastases were surgically treated, and diagnosed by pathological examination of resected specimens. In two cases, metastasis was diagnosed using endoscopic USguided fine-needle aspiration cytology (EUSFNA) [6, 7].

Before we decided to perform the pancreatic tail resection as the definitive surgery, shortly after the successful hemostasis, new lesions were found in her liver and lung. The possibility of metastasis from the previous malignant PT was considered, as were other differential diagnoses, such as primary pancreatic tumor and metastases. We forewent use of diagnostic EUSFNA, as the pancreatic tumor was located in the pancreatic dorsum and was
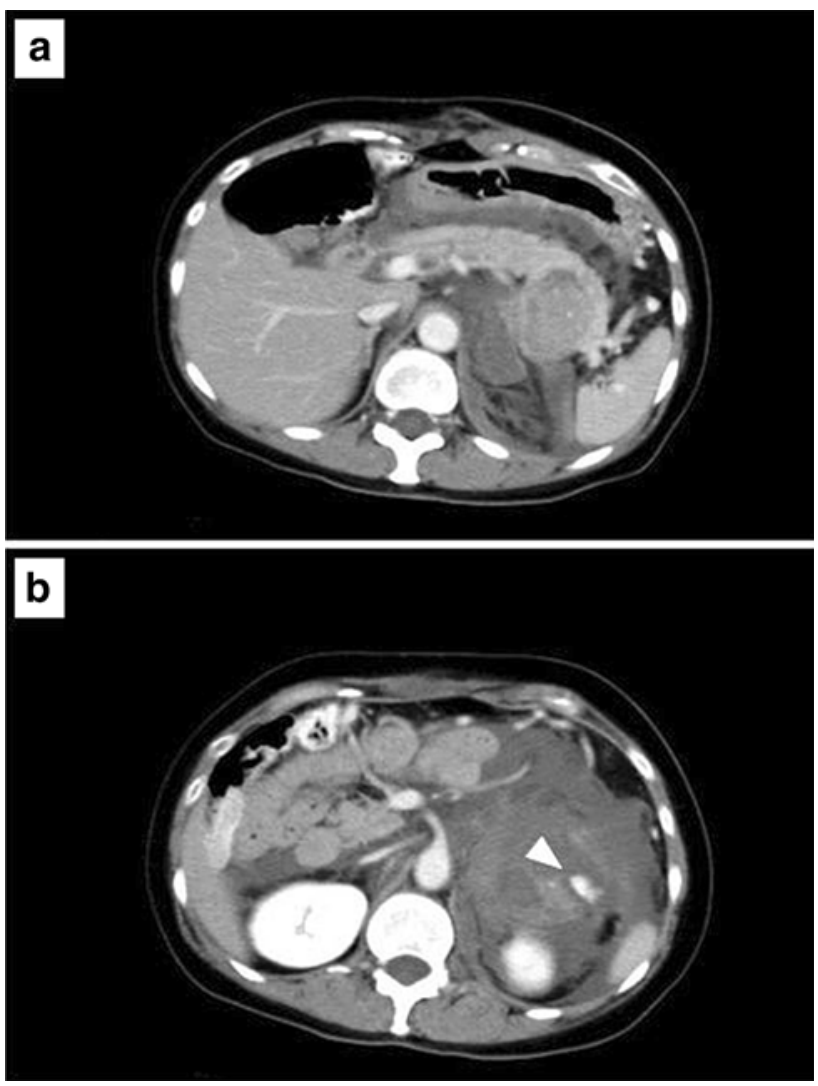

Fig. 3 Enhanced CT upon admission revealed a pancreatic tumor (a) and extravasation of contrast medium (arrowhead) in the retroperitoneal hematoma $(\mathbf{b})$

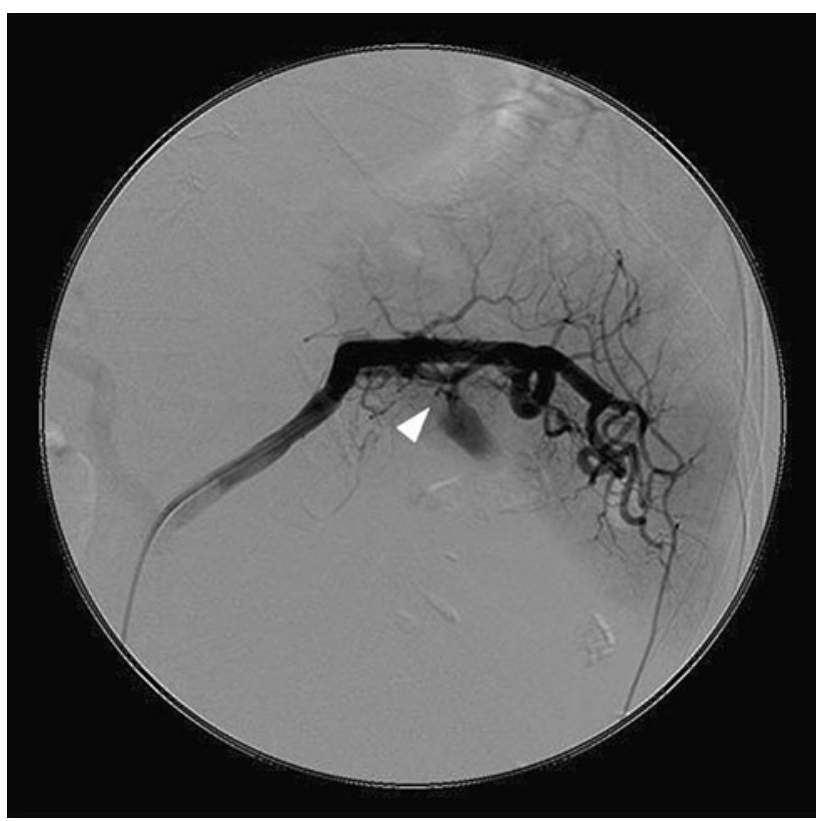

Fig. 4 Urgent angiography revealed a bleeding branch of the splenic artery with contrast extravasation (arrowhead) 

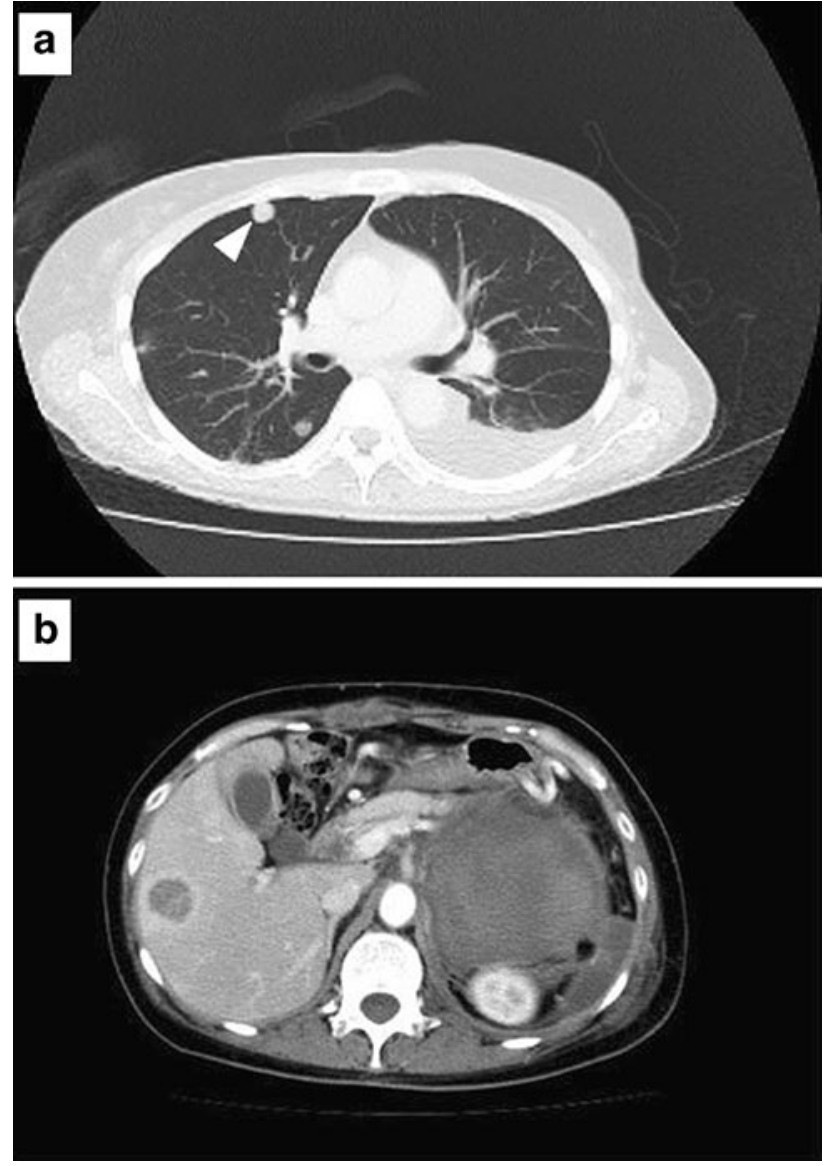

Fig. 5 Follow-up CT 16 days after the embolization disclosed new tumors in the lung (a) and in the liver (b). A lung tumor (arrowhead) was biopsied

associated with a large retroperitoneal hematoma. We performed the less-invasive lung tumor biopsy by VATS instead of pancreatic tail resection. Although we could not obtain direct evidence of pancreatic metastasis, considering the pathology of the lung tumor and the timing of the onset of the pancreas, liver, and lung tumors after the breast surgery, we concluded that the pancreas tumor was the initial metastasis of the breast malignant PT, and that it caused her pain and retroperitoneal hemorrhage as the first presentation of symptoms of distant metastases. To the best of our knowledge, retroperitoneal hemorrhage due to pancreatic metastasis from malignant PT has not been previously documented in the English-language literature.

Metastatic PT of the breast has a poor prognosis; average survival time of patients with PT metastasis is reported to be 30 months [11]. Suzuki-Uematsu et al. [12] reviewed reports of malignant PT with metastases in Japan; seven of 15 reported patients with metastatic PT died within 1 year after resection. Among the four resected cases of pancreatic metastasis from PT [6, 8-10], two cases showed early relapse $[8,9]$, whereas one patient survived for a long time
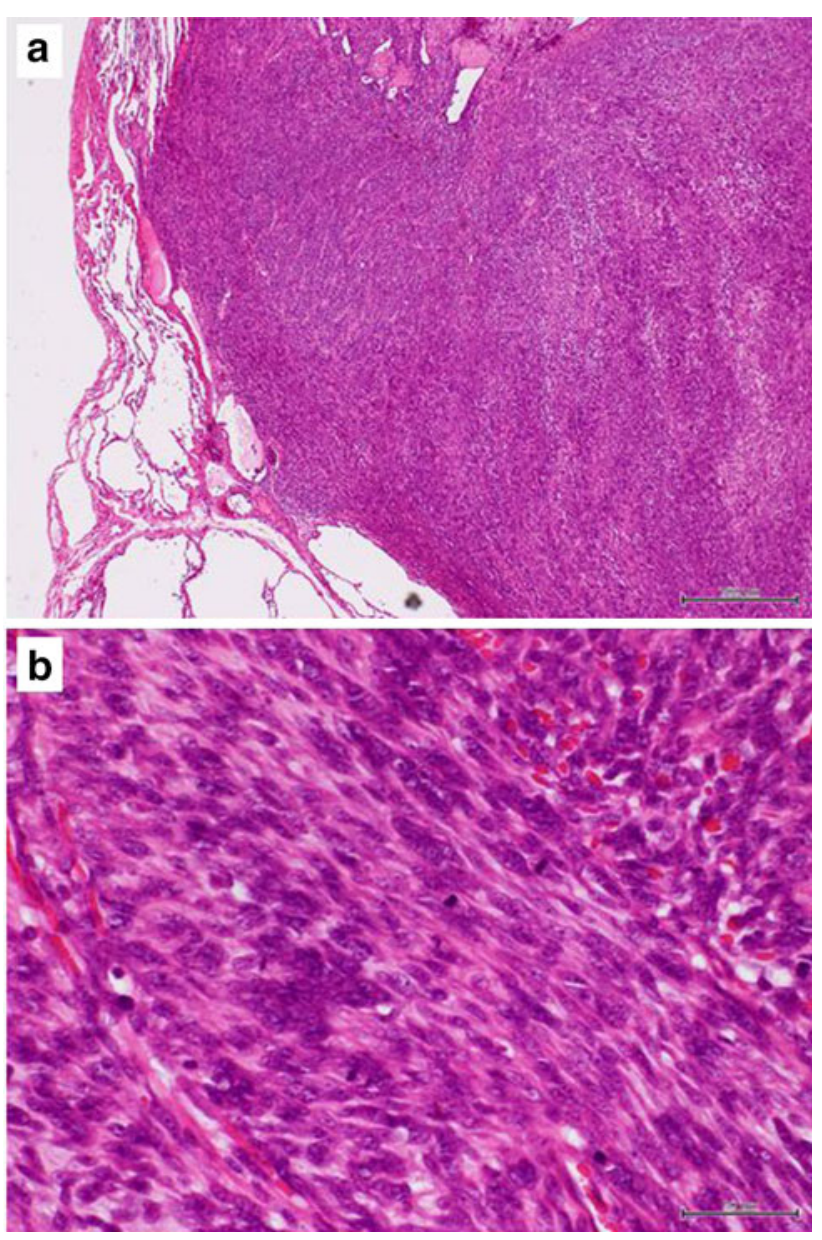

Fig. 6 Microscopic findings of lung metastasis: low-power magnification (a) and high-power magnification (b). Metastatic tumor comprises only stromal component (a) and it mimics the malignant part of primary tumor (b). Scale bars $500 \mu \mathrm{m}$ in $\mathbf{a}$ and $50 \mu \mathrm{m}$ in b

after resection [6]. Because there are few benefits from surgery when new metastases appear in a short time, systemic treatment should be considered early.

Although standard chemotherapy for metastatic PT is not established yet, chemotherapy that includes ifosfamide is reported to be effective. Hawkins et al. [13] reported two complete remission cases; one patient was treated with a combination regimen containing ifosfamide and doxorubicin, and was disease-free for 61 months. Tsuchie et al. [14] reported a case that showed relapsed metastasis to lung and pancreas 3 months after a pancreaticoduodenectomy for intrapancreatic bile duct metastases of malignant PT. For this patient, they used the same combination chemotherapy as Hawkins; the case completely remitted and the patient was disease-free for the next 5 years.

Successful catheter intervention against retroperitoneal hemorrhage from metastatic tumor is also uncommon [15]. Patients under spontaneous retroperitoneal hemorrhage usually require urgent surgical exploration [16]. In our case, 

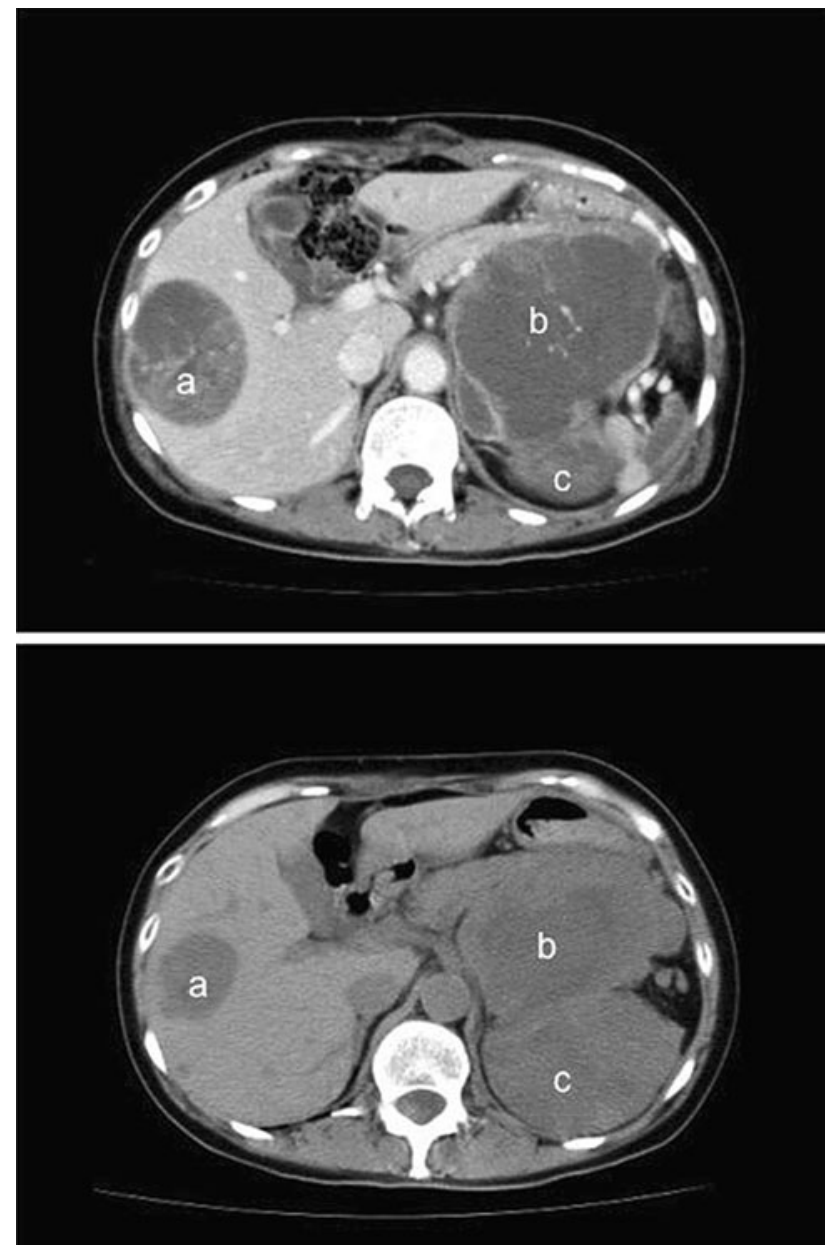

Fig. 7 Five weeks after biopsy, CT before chemotherapy (top) showed rapidly growing liver $(a)$ and pancreatic $(b)$ metastases. After 1 course of chemotherapy (bottom), liver $(a)$ and pancreatic $(b)$ metastases show partial regression; new lesion $(c)$ shows rapid growth

as the exacerbation of symptoms occurred in the hospital, we could detect retroperitoneal hemorrhage early and were able to perform urgent angiography. Because the catheter intervention was successful, we could avoid an urgent exploratory laparotomy and unnecessary highly invasive definitive surgery, and were able to decide on a systemic treatment. The combination regimen containing ifosfamide and doxorubicin was employed in our patient. Although the target lesion was partially remitted, a new lesion developed at the same time. Hemostasis was not simple in our case; the very large retroperitoneal hematoma was left for a long time, which affected our patient's recovery. The malignant PT progressed so quickly that the delayed initiation of chemotherapy may have affected her response to it. There are few reports of PT metastasizing to retroperitoneal spaces such as the pancreas [6-10] or the adrenal gland [17]. However, the patients with metastases in multiple sites could eventually develop retroperitoneal metastasis; this could lead to retroperitoneal hemorrhage, considering the aggressive nature of metastatic PT. We should be careful about the symptoms indicating the sign of bleeding during the follow-up of patients with fast-progressing metastatic PT that quickly develops new metastases.

Conflict of interest The authors declare that they have no conflict of interest.

\section{References}

1. Bellocq JP, Magro G (2003) Fibroepithelial tumours. In: Tavassoli FA, Devilee P (eds) World Health Organization classification of tumours: pathology and genetics tumours of the breast and female genital organs. IARC, Lyon, p 99-103

2. Rosen PP (2009) Fibroepithelial neoplasm. In: Rosen PP (ed) Rosen's breast pathology. Lippincott Williams \& Wilkins, Philadelphia, p 187-229

3. Calhoun KE, Lawton TJ et al (2010) Phyllodes tumors. In: Harris JR, Lippman ME, Morrow M, Osborne CK (eds) Diseases of the breast, 4th edn. Lippincott Williams \& Wilkins, Philadelphia, p 781-792

4. Noguchi S, Aihara T, Motomura K et al (1996) Phyllodes tumor of the breast: pathology, histogenesis, diagnosis, and treatment. Breast Cancer 3:79-92

5. Ward RM, Evans HL (1986) Cystosarcoma phyllodes: a clinicopathologic study of 26 cases. Cancer 58:2282-2289

6. Serikawa M, Sasaki T, Kobayashi K et al (2012) Malignant phyllodes tumor metastatic to the pancreas: a case report (in Japanese with English abstract). Nihon Shokakibyo Gakkai Zasshi. 109:795-803

7. Ang TL, Ng VW, Fock KM et al (2007) Diagnosis of a metastatic phyllodes tumor of the pancreas using EUS-FNA. JOP. 8:35-38

8. Yu PC, Lin YC, Chen HM et al (2000) Malignant phyllodes tumor of the breast metastasizing to the pancreas: case report. Chang Gung Med J 23:503-507

9. Nakahara R, Fukushima H, Suszuki K et al (1986) A case of metastasizing cystosarcoma phyllodes to the pancreas and lung (in Japanese). Rinsho Geka 41:1709-1713

10. Wolfson P, Rybak BJ, Kim U (1978) Cystosarcoma phyllodes metastatic to the pancreas. Am J Gastroenterol 70:184-187

11. Kessinger A, Foley JF, Lemon HM et al (1972) Metastatic cystosarcoma phyllodes: a case report and review of the literature. J Surg Oncol 4:131-147

12. Suzuki-Uematsu S, Shiraishi K, Ito T et al (2010) Malignant phyllodes tumor composed almost exclusively of a fibrosarcomatous component: a case report and review of malignant phyllodes tumors with metastases. Breast Cancer 17:218-224

13. Hawkins RE, Schofield JB, Wiltshaw E et al (1992) Ifosfamide is an active drug for chemotherapy of metastatic cystosarcoma phyllodes. Cancer 69:2271-2275

14. Tsuchie K, Kuriki H, Sakaguchi K et al (2009) A case report of malignant phyllodes tumor of the breast with a rare metastatic site (intrapancreatic bile duct) and treated with effective chemotherapy resulting long CR (in Japanese). Syujutu 63:267-272

15. Ambika S, Melton A, Lee D et al (2009) Massive retroperitoneal adrenal hemorrhage secondary to lung cancer metastasis treated by adrenal artery embolization. Clin Lung Cancer 10:E1-E4

16. Vasinanukorn P, Rerknimitr R, Sriussadaporn S et al (2007) Adrenal hemorrhage as the first presentation of hepatocellular carcinoma. Intern Med 46:1779-1782

17. Shigekawa T, Sato K, Tamaki K et al (2003) A case of metastases of the lung and adrenal gland from malignant phyllodes tumor successfully treated by ifosfamide (in Japanese with English abstract). Nihon Rinsho Geka Gakkai Zasshi 64:1858-1863 